Research Article

\title{
Improvement and Simulation of THOR Formula with Yaw Angle
}

\author{
Jie Wang ${ }^{1}$, Ying-chun Wang ${ }^{1, *}$, Sheng-qing $\mathrm{Zhu}^{2}$ and Wei-hua Ren ${ }^{1}$ and Tian-lin Niu ${ }^{1}$ \\ ${ }^{1}$ Air and Missile Defense College, Air Force Engineering University, Xi'an 710051, China \\ ${ }^{2}$ Department of Offshore Engineering, American Bureau of Shipping, Singapore
}

Received 30 October 2013; Accepted 19 April 2014

\begin{abstract}
The THOR formula is widely used in the investigation of vulnerability and effectiveness of weapon system, however, its application was limited by the small slenderness ratio and limited materials of target. In order to investigate the damage effect of KE-rod warhead, this paper, basing on the classic THOR formula, focused on improving the formula of residual velocity and residual mass. The improved THOR model could predict the residual velocity and residual mass of KE-rod penetration in the complex conditions, and the predictions were found to be consistent with the experimental numerical results in the literatures. As the experimental data is limited, for the better validation of the improved formula, the paper investigated further research and verification of the improvement THOR formula with numerical simulation. Since the experimental data are limited for hypervelocity impact, comparisons of results between M-THOR with experimental and numerical data were widely preceded. The error is less than $4.8 \%$ for the predicted residual velocity while $5.4 \%$ for predicted residual velocity. The effect of yaw angle in the modified THOR formula was also found to agree quite well with the reference.
\end{abstract}

Keywords: penetration, projectile, target, KE-rod, yaw angle, improvement, calculation

\section{Introduction}

KE-rod warhead technology offers designers a novel warhead that can attack and destroy the ballistic missiles $[1,2,3]$. By contrast with the traditional projectiles and long rod penetrators, the KE-rod is well known of high density, great slenderness ratio, high flying velocity, etc. $[3,4,5]$. Researches on the residual velocity and mass of killing elements have found to be of great significance on the residual power and damage assessment.

Detailed analyses of the penetration characteristics of cylindrical projectiles have included the effect of the slenderness (L/D) ratio in the penetration models by Anderson et al. [6] and Chocron et al. [7]. In particular, the work done by Anderson et al. [8] revealed that numerical results tended to be in very good agreement with experiment data. Researches by Johnson and Cook [9] and by Gee and Littlefield [10] demonstrated that numerical simulations provided reasonably accurate results for impact events involving cylindrical projectiles, at various obliquities, yaw angles, and impact velocities. However, the range of obliquity and yaw angles used in their investigations is relatively limited.

Chen and $\mathrm{Li}$ [11] proposed an impact function and the geometry function of a projectile, two dimensionless parameters used to formulate the critical impact limit at the transition from non-deformable projectile penetration regime to semi-hydrodynamic penetration regime. They observed good agreement between experimental results and theoretical predictions using different materials in a broad

*E-mail address: wyc1986@163.com ISSN: 1791-2377@ 2014 Kavala Institute of Technology. All rights reserved. range of impact velocities and projectile geometries.

Taylor and Tate [12] modified the material strength of projectile and target, and obtained the mass erosion law of long rod penetrators impacting on the target vertically with the fluid dynamics theory. Gunnar W. Recht and Yatteau [13] revised the traditional Light of Sight Model, and established the High Obliquity Model for long rod penetrator impacting on the plate obliquely. Furthermore, Gunnar W. Recht and Yatteau created the residual mass model of finite plate based on the improved Tate Model, which was used in the endgame analyzes procedure FATEPEN2 [14] and had better accuracy.

The THOR formula [15] was a statistical fitting of a large number of experimental data based on projectiles with various materials and shapes for the prediction of the ballistic resistance with projectile penetrating the armoured material, which was widely used in the analysis of vulnerability and effectiveness of weapon system as the high accuracy for prediction [16], however, its application is limited by the following conditions: (1) the slenderness ration of projectile is less than 3 ; (2) the projectiles don't broke up when penetrating; (3) the obliquity angle $\theta_{R}$ must be in the range of $70^{\circ}$; (4) the material of projectile should be in the Table 1.

Lu Y.-G., and Yang S.-Q. modified the THOR formula to apply in the condition of large slenderness ratio, great obliquity angle and high yaw angle. The paper focuses on the THOR formula in the work of literature [17]. The improved THOR formula could better reflect the real situation in term of the collision, and accurately calculate the relationships among the yaw angle and the residual mass, the yaw angle and the residual velocity. 


\section{THOR Formula Model of KE-rod}

\subsection{Original THOR Formula Model}

The basic formula of original THOR model contains: the residual velocity THOR formula, the protective velocity THOR formula of target and the residual mass THOR formula. The residual velocity THOR formula and the residual mass THOR formula concerned by the paper is [17]

$$
\begin{gathered}
V_{r}=V_{R}-0.3048 \times 10^{c_{11}}(61023.75 h A)^{c_{12}} \\
\quad\left(15432.1 m_{0}\right)^{c_{13}}\left(\sec \theta_{R}\right)^{c_{14}}\left(3.28084 V_{R}\right)^{c_{15}} \\
M_{r}=m_{s}-6.48 \times 10^{c_{31}-5}(61023.75 h A)^{c_{32}} \\
\quad\left(15432.1 m_{0}\right)^{c_{33}}\left(\sec \theta_{R}\right)^{c_{34}}\left(3.28084 V_{R}\right)^{c_{35}}
\end{gathered}
$$

where $\quad V_{r}$ - residual velocity of projectile $(\mathrm{m} / \mathrm{s})$;

$V_{R}$ - penetration velocity of projectile $(\mathrm{m} / \mathrm{s})$;

$h$ - thickness of plate $(\mathrm{m})$;

$A-$ contact area of projectile and plate $\left(\mathrm{m}^{2}\right)$;

$m_{0}$ - initial mass of projectile $(\mathrm{kg})$;

$M_{r}$ - residual mass of projectile $(\mathrm{kg})$;
$\theta_{R}$ - cross angle of velocity vector and the normal of plate (illustrated in Fig. 1);

$c_{11} \sim c_{35}$ - constants or coefficients of materials for target defined in the THOR formula (shown in Tab. $1[18])$.

\begin{tabular}{|c|c|c|c|c|c|c|c|c|c|c|}
\hline Material & $c_{11}$ & $c_{12}$ & $c_{13}$ & $c_{14}$ & $c_{15}$ & $c_{31}$ & $c_{32}$ & $c_{33}$ & $c_{34}$ & $c_{35}$ \\
\hline Magnesium & 6.904 & 1.092 & -1.170 & 1.050 & -0.087 & -5.945 & 0.285 & 0.803 & -0.172 & 1.519 \\
\hline Aluminum 2024-T3 & 7.074 & 1.029 & -1.072 & 1.251 & -0.139 & -6.663 & 0.227 & 0.694 & -0.316 & 1.901 \\
\hline Titanium & 6.292 & 1.103 & -1.095 & 1.369 & 0.167 & 2.318 & 1.086 & -0.748 & 1.327 & 0.459 \\
\hline Cast Iron & 4.840 & 1.042 & -1.051 & 1.028 & 0.523 & -9.703 & 0.162 & 0.673 & 2.091 & 2.710 \\
\hline Mild Steel & 4.356 & 0.674 & -0.791 & 0.989 & 0.434 & 1.195 & 0.234 & 0.744 & 0.469 & 0.483 \\
\hline RHA Armor & 6.399 & 0.889 & -0.945 & 1.262 & 0.019 & -2.507 & 0.138 & 0.835 & 0.143 & 0.761 \\
\hline FH Armor & 6.475 & 0.889 & -0.945 & 1.262 & 0.019 & -2.264 & 0.346 & 0.629 & 0.327 & 0.880 \\
\hline Copper & 2.875 & 0.678 & -0.730 & 0.846 & 0.802 & -5.489 & 0.340 & 0.568 & 1.422 & 1.650 \\
\hline Lead & 1.999 & 0.499 & -0.502 & 0.655 & 0.818 & -1.856 & 0.506 & 0.350 & 0.777 & 0.934 \\
\hline Tuballoy & 2.537 & 0.583 & -0.603 & 0.865 & 0.828 & -3.379 & 0.560 & 0447 & 0.640 & 1.381 \\
\hline Nylon Unbonded & 5.816 & 0.835 & -0.654 & 0.990 & -0.162 & -7.538 & -0.067 & 0.903 & -0.351 & 0.717 \\
\hline Nylon Bonded & 4.672 & 1.144 & -0.968 & 0.743 & 0.392 & -13.601 & 0.035 & 0.775 & 0.045 & 3.451 \\
\hline Lexan & 2.908 & 0720 & -0.657 & 0.773 & 0.603 & -0.275 & 0.480 & 0.465 & 1.171 & 1.765 \\
\hline Plexiglass Cast & 5.243 & 1.044 & -1.035 & 1.073 & 0.242 & -2.342 & 1.402 & -0.137 & 0.674 & 1.324 \\
\hline Plexiglass Stretched & 3.605 & 1.112 & -0.903 & 0.715 & 0.686 & -5.344 & 0.437 & 0.169 & 0.620 & 1.683 \\
\hline Doron & 7.600 & 1.021 & -1.014 & 0.917 & -0.362 & -10.404 & 0.215 & 0.343 & 0.706 & 2.906 \\
\hline Bullet Resistant Glass & 3.743 & 0.705 & -0.723 & 0.690 & 0.465 & -5.926 & 0.305 & 0.429 & 0.747 & 1.819 \\
\hline
\end{tabular}

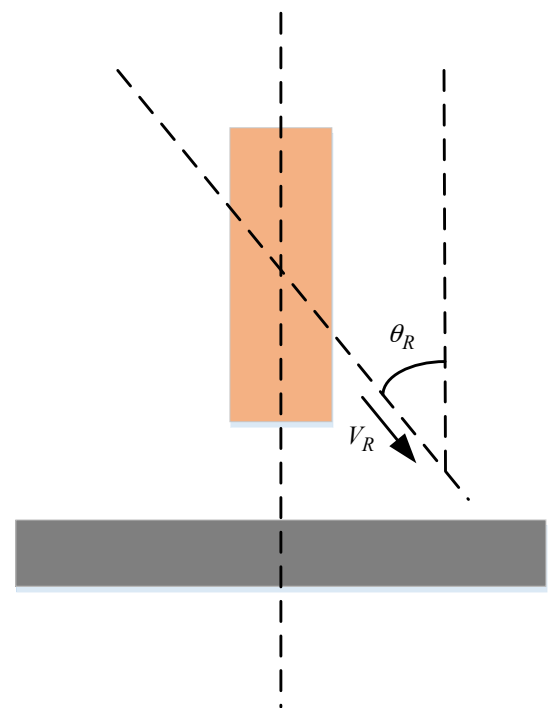

Fig. 1 Diagram of $\theta_{R}$ and plate

Table 1. Constants of material for THOR formula

As the THOR formula is obtained by fitting the experimental data, during the application of THOR formula in last decades, it is widely concluded by its uses that, application of THOR formula is limited in the following regions:

(1) The slenderness ratio of projectile is not more than 3;

(2) The materials of target offered by the experiment are limited, the other materials should be derived from the materials of experiment, that is to say, selecting the strength of material close to the material with experiment, then, modified the thickness of target by contrast of the density of unknown material and the material used in THOR formula;

(3) The THOR formula is used when the projectiles are not fracture in term of penetration;

(4) The oblique angle should be in the range of $0^{\circ}-70^{\circ}$, the projectile is invalid when the oblique angle goes beyond $70^{\circ}$;

(5) The material of projectile should be in the Table 1.

\subsection{THOR Formula for KE-rod Vertical Penetration on Target}

Reference [17] finds that: the THOR formula is not applicable to calculate the residual mass of KE-rod when the slenderness ratio is more than 3 , and the residual velocity is not affected by the limit of the slenderness ratio. The theoretical analysis and experiments of mass loss mechanism for long rod penetration on the target show that [17]: as the thickness of target and velocity of rod is constant, the mass loss of rod has a maximum value, that is to say, the mass loss increases with the slenderness ratio slowly, when the slenderness ratio reaches a certain value, the mass loss or rod reaches the maximum. On the other hand, according to the impact dynamics theory, the mass loss of rod is due to the plastic waves induced by the head of rod impact on target for the plate penetration, the effect begins from the time of rod touching the plate, and ends with the counteracting the plastic waves of rod impact on the plate and the rarefaction waves that reflected back from the plate bottom. So the 
thickness of rod and the elastic velocity of rod an plate have an important influence on the residual mass of the rod, the residual mass of the rod was modified, and the improved THOR formula is

$$
\begin{aligned}
V_{r}= & V_{R}-0.3048 \times 10^{c_{11}}(61023.75 h A)^{c_{12}} \\
& \left(15432.1 m_{0}\right)^{c_{13}}\left(\sec \theta_{R}\right)^{c_{14}}\left(3.28084 V_{R}\right)^{c_{15}} \\
M_{r}= & m_{s}-6.48 \times 10^{c_{31}-5}(61023.75 h A)^{c_{32}} \\
& \left(15432.1 \cdot F_{m} m_{0}\right)^{c_{33}}\left(\sec \theta_{R}\right)^{c_{34}}\left(3.28084 V_{R}\right)^{c_{35}} \\
F_{m}= & \frac{2}{\pi} \arctan \left(\frac{l}{h}\right)\left(\frac{5 C_{e p}}{C_{e t}} \cdot \frac{h}{l}\right)
\end{aligned}
$$

where $\quad C_{e p}-$ elastic velocity of projectile;

$$
C_{e t} \text { - elastic velocity of target; }
$$$$
l \text { - initial length of the rod. }
$$

\section{Improvement of THOR Formula with Yaw Angle}

The classical THOR formula considers the initial velocity, the obliquity angle, the mass of the projectile, the thickness of target and the contact area of penetration, ignoring the effect of the yaw angle, that is, the formulas is only capable of estimating the residual velocity and mass of KE-rod when the yaw angle is $0^{\circ}$. In practise, even if the above five factors are identical, the residual velocity will change with the yaw angle. Therefore, the effect of the yaw angle should be considered in the actual application, and it's necessary to modify the THOR formulas with the yaw angle. Fig. 2 shows the KE-rod penetration on plate with yaw angle $\beta$.

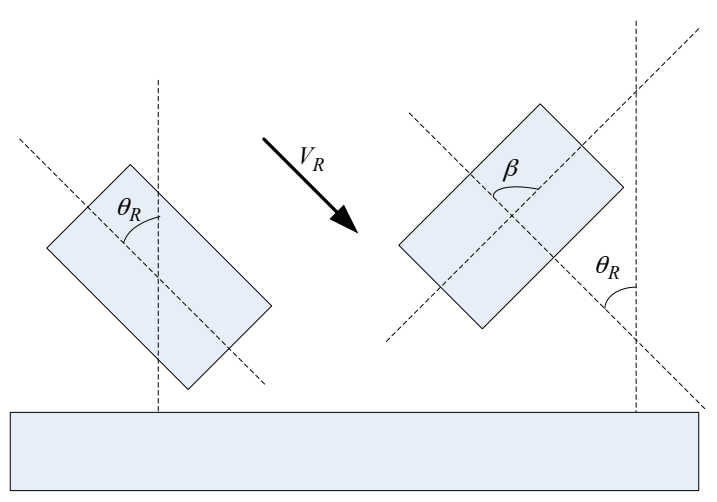

Fig. 2 Diagram of KE-rod penetration with yaw angle

We transform the model of KE-rod with the yaw angle into the projection area of KE-rod in the plate, the process of simplified model is shown in Fig. 3.

(1) As the rod is the cylinder, and the reference [17] takes the rod as the equivalent cuboid rod, so there are some errors of the equivalent area in the real situation. The paper takes the KE-rod as the equivalent elliptical rod, which meets the actual circs better, and ignores the width difference of the equivalent rod and the original, and supposes that the length of penetration is the same.

(2) The equivalent projection area contains two semiellipse areas and a rectangular area, which will lead to better accuracy comparing with the reference [17].

$$
A^{\prime}=\pi l_{2} \frac{d}{2}+d l_{1}
$$

$$
l_{1}=l \sin \beta
$$

$$
l_{2}=\frac{d}{2} \cos \beta
$$

where $\quad A^{\prime}$-projection area of KE-rod $\left(\mathrm{m}^{2}\right) ;$
$l$-length of the KE-rod $(\mathrm{m}) ;$

$d$-diameter of the KE-rod.

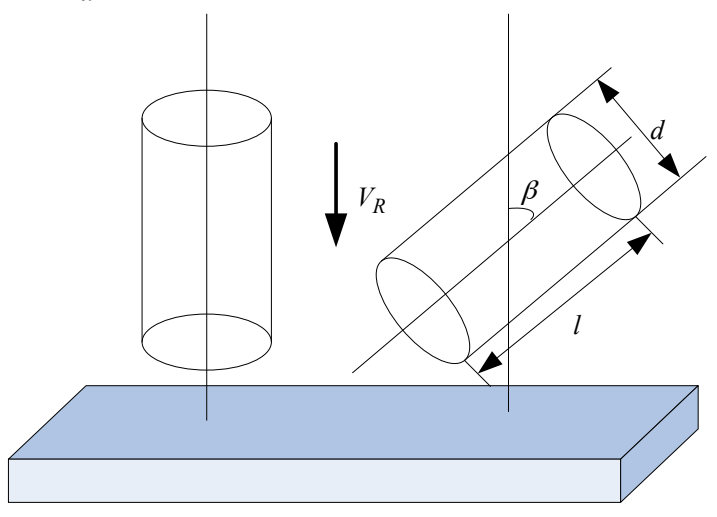

(a)

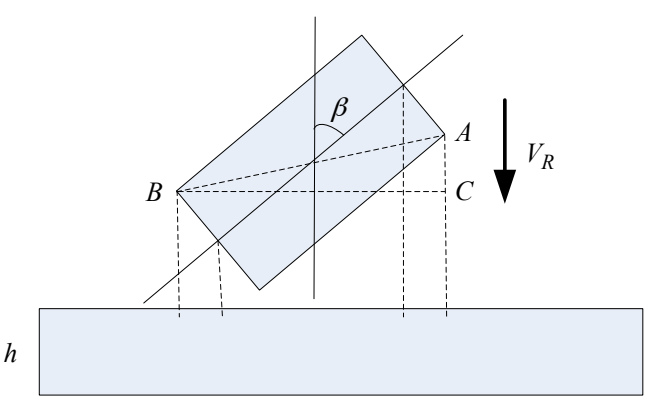

(b)

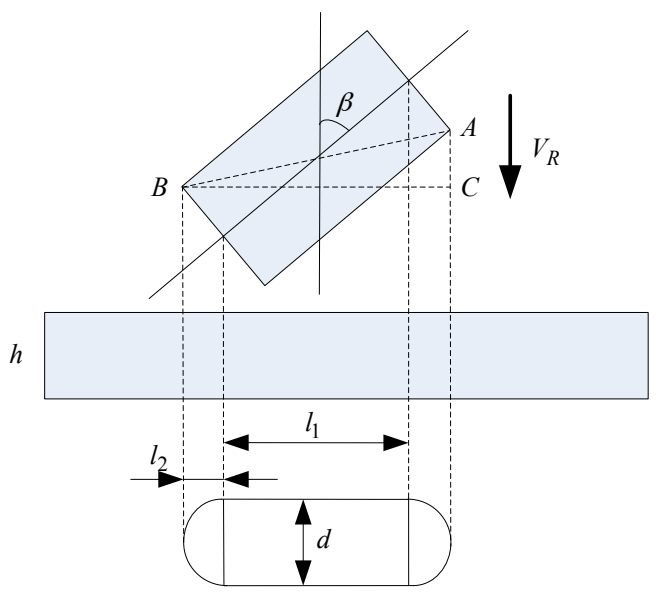

(c)

Fig. 3 Calculation of projection area for KE-rod

The residual velocity and residual mass of the modified THOR formulas with yaw angle are given by 


$$
\begin{aligned}
V_{r}= & V_{R}-0.3048 \times 10^{c_{11}}\left(61023.75 h A^{\prime}\right)^{c_{12}} \\
& \left(15432.1 m_{0}\right)^{c_{13}}\left(\sec \theta_{R}\right)^{c_{14}}\left(3.28084 V_{R}\right)^{c_{15}} \\
M_{r}= & m_{S}-6.48 \times 10^{c_{31}-5}\left(61023.75 h A^{\prime}\right)^{c_{32}} \\
& \left(15432.1 \cdot F_{m} m_{0}\right)^{c_{33}}\left(\sec \theta_{R}\right)^{c_{34}}\left(3.28084 V_{R}\right)^{c_{35}}
\end{aligned}
$$

\section{Numerical Simulations and Discussion}

\subsection{Verification with Experimental Data}

The paper simulated the relationship between yaw angle and the changes of mass loss with yaw angle based on the aforementioned modified THOR formula comparing with the experimental data in reference [14].

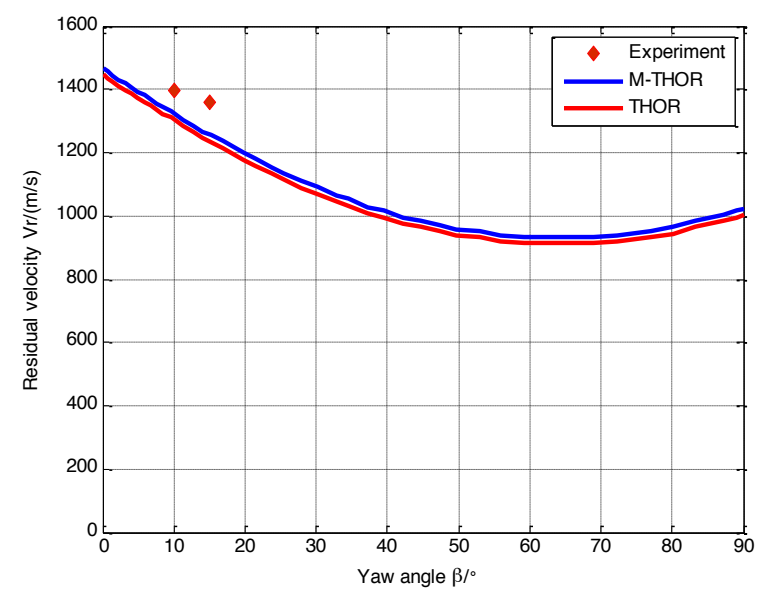

Fig. 4 Comparison of residual velocity with experiment

\subsection{Further Investigation of Modified THOR Formula}

In order to validate the relationship among the yaw angle and the residual velocity and mass comprehensively, the paper verifies the modified THOR formula with numerical simulation. The initial penetration condition is the same as the reference $[19,20]$. The paper simulates the KE-rod penetrating on the plate with the software ANSYS/LSDYNA to validate the modified THOR formula.

The rod material is $20 \#$ steel, diameter $d$ is $0.01 \mathrm{~m}$, and the slenderness ratio $l / d$ is 10 , the initial mass is $61.7 \mathrm{~g}$; the
Fig. 4 shows the relationship between residual velocity and yaw angle, it can be found that the residual velocity decreases as the yaw angle increasing, and the residual velocity of modified THOR formula is marginally more accurate than the THOR formula; Fig. 5 reflects the changes of mass loss with the yaw angle that the mass loss increases with yaw angle and the mass loss of modified THOR formula is distinctly more accurate than the THOR formula obviously.

As the data of experiment is limited, the data couldn't verify the modified THOR formula comprehensively. According to the trend of changes of residual velocity and mass loss with yaw angle, the error of residual velocity and mass loss compared modified THOR formula with experiment is less than the THOR formula, in other words, the results of modified THOR formula is better than the original THOR formula.

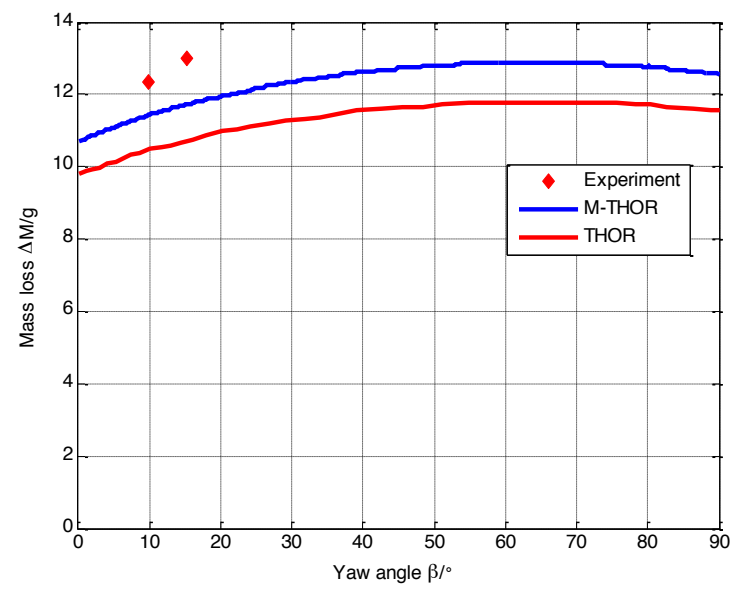

Fig. 5 Comparison of mass loss with experiment

plate material is duralumin, and the thick is $0.01 \mathrm{~m}$ and $0.02 \mathrm{~m}$. The initial velocities of rod are $800 \mathrm{~m} / \mathrm{s}$ and $2000 \mathrm{~m} / \mathrm{s}$, the yaw angle $\beta$ takes value $10^{\circ}, 30^{\circ}, 50^{\circ}, 70^{\circ}$ and $90^{\circ}$. The simulation model was established with the software ANSYS/LS-DYNA, the Johnson-Cook constitutive model [21] and Mie-Gruniesen equation of state [22] were taken into account thermal softening, strain hardening, and strain rate effects on the plastic flow regime for metals. The

\begin{tabular}{|c|c|c|c|c|c|c|c|c|c|}
\hline Material & $\begin{array}{c}\text { Yield } \\
\text { stress/MPa }\end{array}$ & $\begin{array}{c}\text { Hardening } \\
\text { constant/MPa }\end{array}$ & $\begin{array}{c}\text { Hardening } \\
\text { exponent }\end{array}$ & $\begin{array}{c}\text { Strain rate } \\
\text { constant }\end{array}$ & $\begin{array}{c}\text { Temperature } \\
\text { exponent }\end{array}$ & $\begin{array}{c}\text { Sound } \\
\text { velocity } /(\mathrm{m} / \mathrm{s})\end{array}$ & $S_{1}$ & $\gamma$ & $A$ \\
\hline Rod & 245 & 426 & 0.36 & 0.014 & 1.05 & 5170 & 1.92 & 1.84 & 0.5 \\
\hline Plate & 275 & 343 & 0.3 & 0.01 & 1.0 & 5090 & 1.34 & 1.68 & 0.5 \\
\hline
\end{tabular}
material parameters of rod and plate are shown in Tab. 2 . The results of numerical simulations are presented in Tab. 3 .

Table 2. Material parameters of rod and plate

The penetrations of KE-rods on plates with different yaw angles are presented in Fig. 6. The failure mode of plate is mainly due to the cutting effect and the penetration effect of KE-rod. At the same yaw angle and initial velocity, the bending of KE-rod and the damage of plate are more obvious as the plate is more thicker. And at the initial velocity of $800 \mathrm{~m} / \mathrm{s}$ and $2000 \mathrm{~m} / \mathrm{s}$, the kinetic energy of the former is much smaller than the latter, at the same yaw angle and thickness of plate, the former is obvious in the bending of KE-rod, and the latter is obvious in the damage of pltae.
On consideration of analytical convenience and visualization, the contents of Tab. 3 are converted to Fig. 6, Fig. 8 and Fig. 9.

Fig. 7 shows the changes of residual velocity with yaw angle. As the initial velocity is $800 \mathrm{~m} / \mathrm{s}$, the residual velocity decreases with the increasing of yaw angle, which reach the minimum value in condition of yaw angle $70^{\circ}$, and then the residual velocity increases slightly; when the thickness of plate is $0.02 \mathrm{~m}$, the residual velocity is almost the limit penetration velocity. As the initial velocity is $2000 \mathrm{~m} / \mathrm{s}$, the 
Jie Wang, Ying-chun Wang, Sheng-qing Zhu and Wei-hua Ren and Tian-lin Niu

.Journal of Engineering Science and Technology Review 7 (2) (2014) $106-112$

residual velocity decreases as yaw angle increases. The reason is that when the rods penetrate on the plates with different yaw angles (except for $\beta=90^{\circ}$ ), in a certain range, with the yaw angle increasing, the velocity decreasing, and the thickness of plate increasing, the attitude change rod is obvious, at the same time, as the initial veolocty is decreasing, the time of penetration is longer, then the effect on the residual velocity is greater.

Table 3. Results of numerical simulation for KE-rod penetration with yaw angle

\begin{tabular}{|c|c|c|c|c|c|c|c|}
\hline \multirow{2}{*}{$\beta\left({ }^{\circ}\right)$} & \multirow{2}{*}{$V_{R}(\mathrm{~m} / \mathrm{s})$} & $V_{r}(\mathrm{~m} / \mathrm{s})$ & $M_{r} / M_{0}$ & Deflectable angle $\left({ }^{\circ}\right)$ & $V_{r}(\mathrm{~m} / \mathrm{s})$ & $M_{r} / M_{0}$ & Deflectable angle $\left({ }^{\circ}\right)$ \\
\hline & & \multicolumn{3}{|c|}{$\mathrm{H}=0.01 \mathrm{~m}$} & \multicolumn{3}{|c|}{$\mathrm{H}=0.02 \mathrm{~m}$} \\
\hline 10 & 800 & 760.3 & 0.945 & 4.6 & 689.7 & 0.891 & 7.3 \\
\hline 10 & 2000 & 1972.6 & 0.922 & 1.4 & 1933.5 & 0.792 & 2.6 \\
\hline 30 & 800 & 695.8 & 0.947 & 9.9 & 548.6 & 0.752 & 23.1 \\
\hline 30 & 2000 & 1867.9 & 0.833 & 6.1 & 1720.2 & 0.543 & 11.2 \\
\hline 50 & 800 & 621.5 & 0.831 & 9.5 & 279.4 & 0.755 & 17.9 \\
\hline 50 & 2000 & 1719.4 & 0.657 & 7.8 & 1332.1 & 0.348 & 12.7 \\
\hline 70 & 800 & 538.3 & 0.927 & 6.4 & 5.3 & - & - \\
\hline 70 & 2000 & 1579.3 & 0.731 & 4.3 & 1112.5 & 0.273 & 10.7 \\
\hline 90 & 800 & 539.2 & 0.956 & - & 126.3 & 0.846 & - \\
\hline 90 & 2000 & 1564.1 & 0.658 & - & 1023.2 & 0.251 & - \\
\hline
\end{tabular}
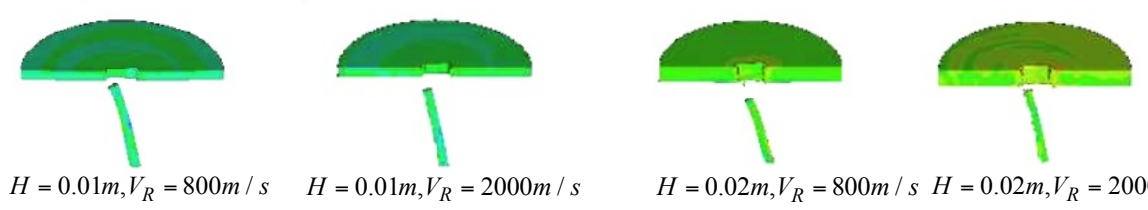

$H=0.01 \mathrm{~m}, V_{R}=2000 \mathrm{~m} / \mathrm{s}$

$H=0.02 \mathrm{~m}, V_{R}=800 \mathrm{~m} / \mathrm{s} \quad H=0.02 \mathrm{~m}, V_{R}=2000 \mathrm{~m} / \mathrm{s}$
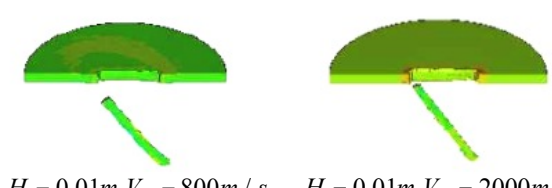

(a) $\beta=10^{\circ}$

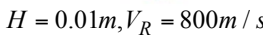

$H=0.01 \mathrm{~m}, V_{R}=2000 \mathrm{~m} / \mathrm{s}$
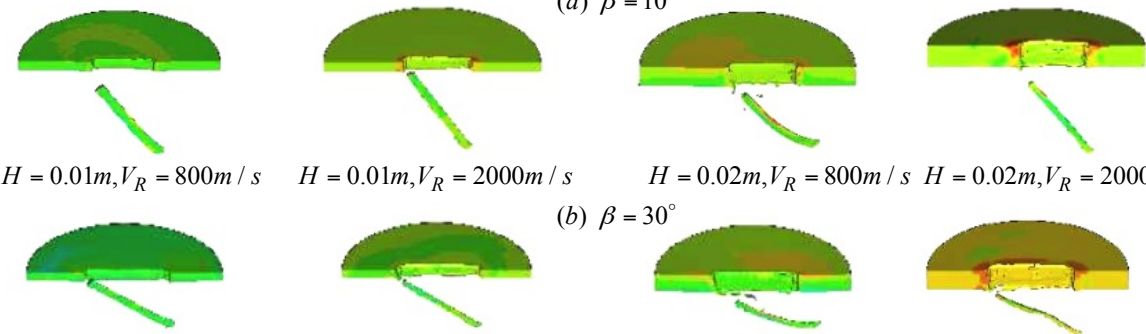

(b) $\beta=30^{\circ}$
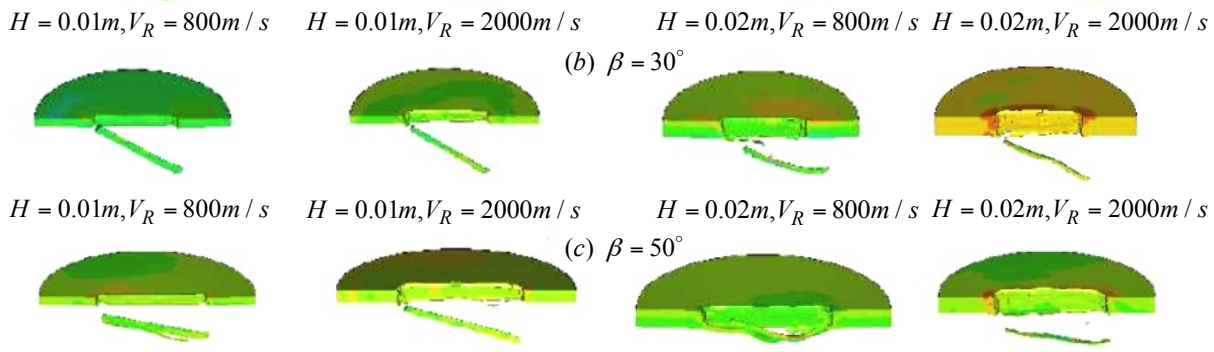

$H=0.01 \mathrm{~m}, V_{R}=2000 \mathrm{~m} / \mathrm{s}$
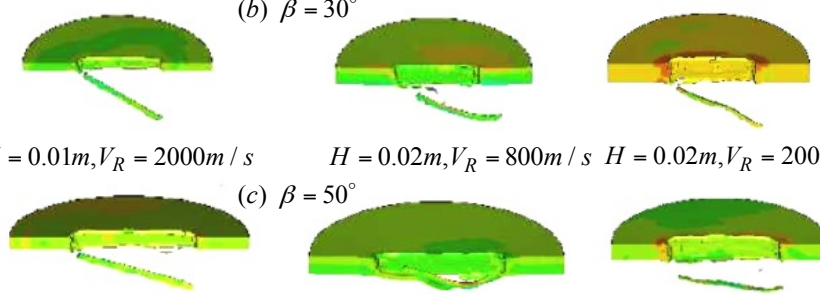

$H=0.02 \mathrm{~m}, V_{R}=800 \mathrm{~m} / \mathrm{s} \quad H=0.02 \mathrm{~m}, V_{R}=2000 \mathrm{~m} / \mathrm{s}$

(c) $\beta=50^{\circ}$
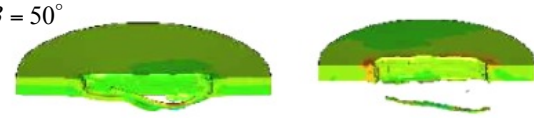

$H=0.01 \mathrm{~m}, V_{R}=800 \mathrm{~m} / \mathrm{s}$

$H=0.01 \mathrm{~m}, V_{R}=2000 \mathrm{~m} / \mathrm{s}$

$H=0.02 \mathrm{~m}, V_{R}=800 \mathrm{~m} / \mathrm{s} \quad H=0.02 \mathrm{~m}, V_{R}=2000 \mathrm{~m} / \mathrm{s}$
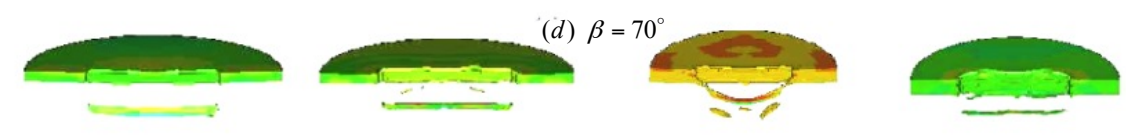

$H=0.01 \mathrm{~m}, V_{R}=800 \mathrm{~m} / \mathrm{s} \quad H=0.01 \mathrm{~m}, V_{R}=2000 \mathrm{~m} / \mathrm{s}$ (d) $\beta=90^{\circ}$

$H=0.02 \mathrm{~m}, V_{R}=800 \mathrm{~m} / \mathrm{s} \quad H=0.02 \mathrm{~m}, V_{R}=2000 \mathrm{~m} / \mathrm{s}$

Fig. 6 Numerical simulation of KE-rod penetration with different yaw angles

The relationship between relative residual mass and yaw angle is shown in Fig. 8, the relative mass decreases with the increasing of yaw angle except the situation $H=0.01 \mathrm{~m}$, $V_{R}=800 \mathrm{~m} / \mathrm{s}$. As the thickness of plate is $0.02 \mathrm{~m}$, the yaw angle is $90^{\circ}$, and the initial velocity is $2000 \mathrm{~m} / \mathrm{s}$, the destruction of KE-rod reaches the maximum, and the residual mass of rod is only about $14.1 \mathrm{~g}$, less than $25 \%$ of the initial mass of the rod. However, as the thickness of plate is $0.01 \mathrm{~m}$, the yaw angle is $90^{\circ}$, and the initial velocity is $800 \mathrm{~m} / \mathrm{s}$, the destruction of rod is minimum, and the residual mass if $58.9 \mathrm{~g}$, the loss of mass is less than $5 \%$ of the initial mass of rod.

Fig. 9 reflects the relationship between deflectable angle and yaw angle, the deflectable angle increases with the yaw angle firstly, and reaches a maximum, then decreases with the increasing of yaw angle.

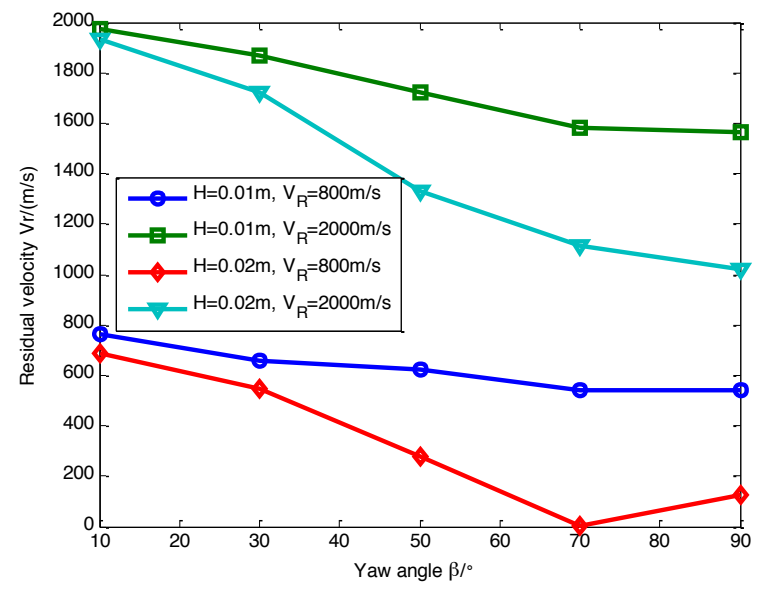

Fig. 7 Relationship of $V_{r}$ and $\beta$ 


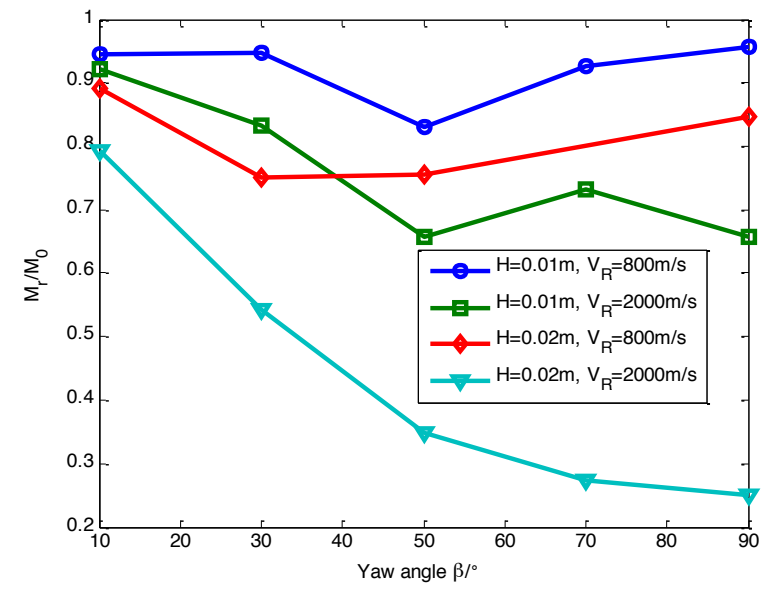

Fig. 8 Relationship of $M_{r} / M_{0}$ and $\beta$

\subsection{Comparison between M-THOR Formula with Numerical Results}

For the valid of modified THOR formula, the paper takes simulation for the initial velocity of KE-rod $2000 \mathrm{~m} / \mathrm{s}$, and the yaw angle $10^{\circ}, 30^{\circ}, 50^{\circ}, 70^{\circ}$ and $90^{\circ}$ penetration on the plates respectively. Fig. 10 shows the numerical simulation

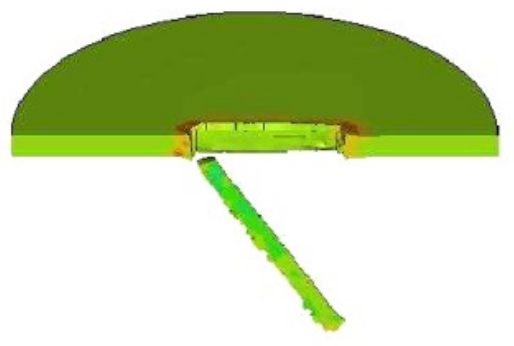

$$
\beta=30^{\circ}, V_{R}=2000 \mathrm{~m} / \mathrm{s}
$$

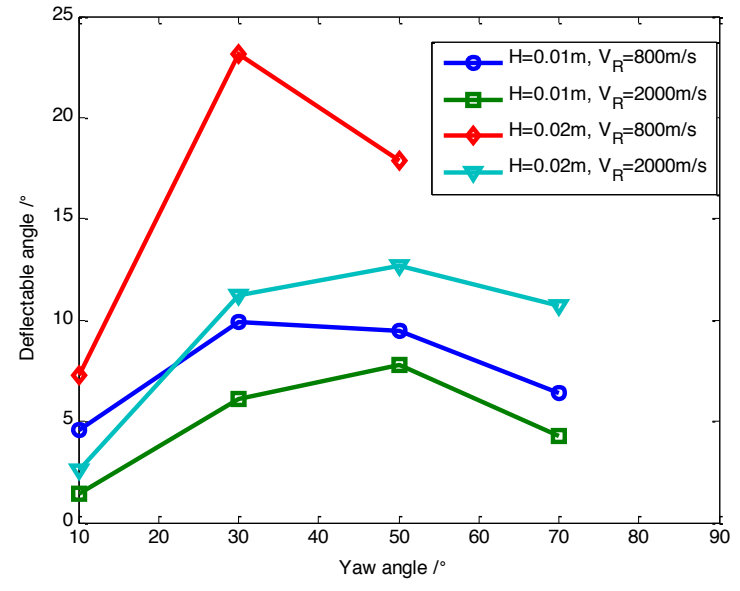

Fig. 9 Relationship of deflectable angle and $\beta$

of rod penetrating on the plate with the yaw angle $\beta\left(30^{\circ}\right.$ and $90^{\circ}$ ). The results of residual velocity and mass from the modified THOR formula comparing with the data from the reference can be found in Fig. 11 and Fig. $12[18,19]$.

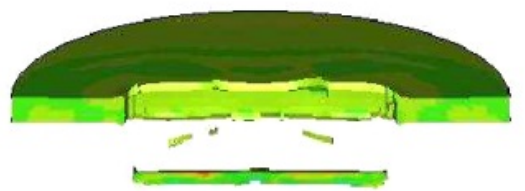

$\beta=90^{\circ}, V_{R}=2000 \mathrm{~m} / \mathrm{s}$

Fig.10 Numerical simulation of rod penetrating on plate with yaw angle $\left(30^{\circ}\right.$ and $\left.90^{\circ}\right)$

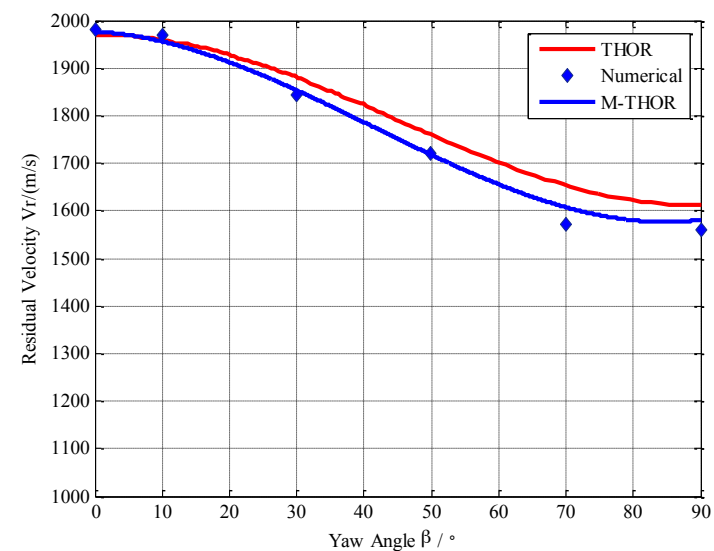

Fig. 11 Relationship of residual velocity and yaw angle

The cutting effect of rod penetration on the plate with high yaw angle is described in Fig. 10 , and the rod doesn't deform with high velocity penetration. The relationship between the residual mass and velocity changing with the yaw angle is illustrated in Fig. 11 and Fig. 12. It can be seen from Fig. 11 and Fig. 12 that the residual velocity and mass reduce as the yaw angle increases, and the changes are not obvious in the begin and end of the range of $10^{\circ}$. Comparing with the numerical results, the residual velocity and mass of the modified THOR formula are better than the THOR formula in reference [19], the error of residual velocity is

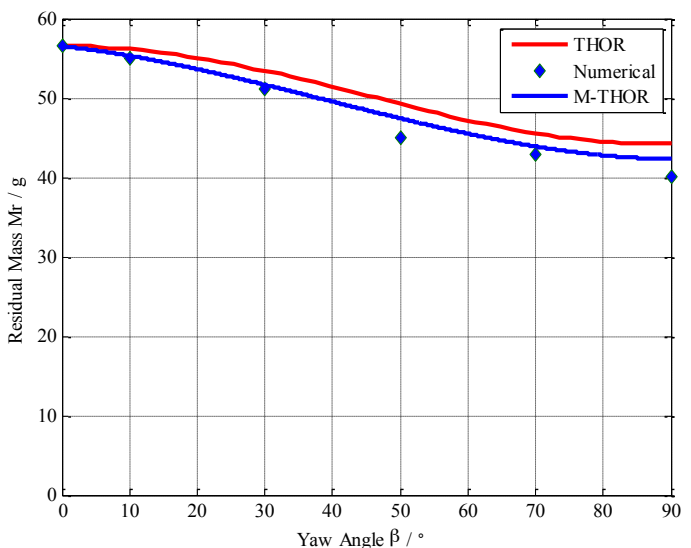

Fig. 12 Relationship of residual velocity and yaw angle

less than $4.8 \%$, and the maximum error of residual velocity is less than $5.4 \%$.

The changes of yaw angle after penetration with the yaw angle are illustrated in Fig. 13, we can see that the changes of yaw angle increase with the yaw angle, then, reach the maximum, and decrease as the yaw angle increases. The maximum change of yaw angle is in the vicinity of $45^{\circ}$. The changes of modified THOR formula agree well with the reference [19]. 


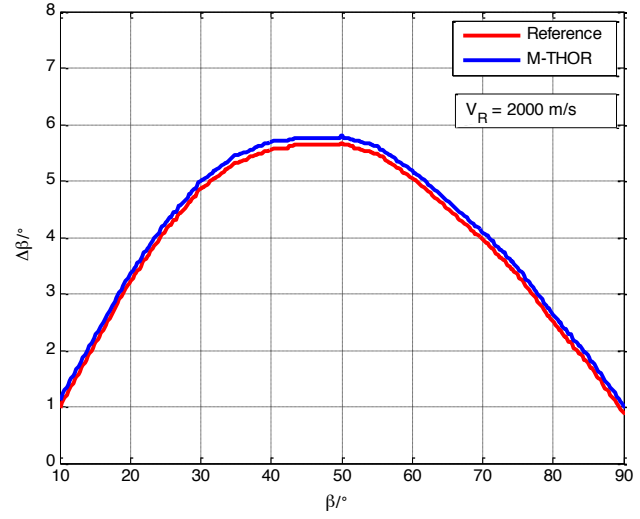

Fig. 13 Changes of yaw angle after penetration with $\beta$

\section{Conclusion}

The THOR formula is improved and calculated based on the reference [17], which contains the residual velocity and the residual mass. When the KE-rod impacts on the target obliquely with the yaw angle, the paper takes the rod as the projection area, and modifies the THOR formula. The modified THOR formula could reflect the relationship among the yaw angle, the residual velocity and the residual mass correctly from the numerical simulations. As the experimental data is limited, for the better validation of the improved formula, the paper investigated further research and verification of the improvement THOR formula with numerical simulation. Since the experimental data are limited for hypervelocity impact, comparisons of results between M-THOR with experimental and numerical data were widely preceded. The error is less than $4.8 \%$ for the predicted residual velocity while $5.4 \%$ for predicted residual velocity. The effect of yaw angle in the modified THOR formula was also found to agree quite well with the reference. The M-THOR formula could provide the reference for the damage assessment of the KE-rod warhead.

\section{References}

1. Wang, Y.-C., Wang, J., Han, B.-B., "Calculation of Kill Probability for Kinetic Energy Rod Against Warhead”, Applied Mechanics and Materials 527, 2014, pp. 100-104.

2. Wang, Y.-C., Wang, J., Du, A.-L., Wang, K., "Damage Indexes of KE-rod Forecast Model Based on BP Neural Network", Systems Engineering and Electronics 35 (9), 2013, pp. 1898-1902. (in Chinese)

3. Lu, Y.-G., Jiang, D.-J., Sui, S.-Y., Li, H.-M., "Experiment study of KE-rod directional dispense", Journal of Ballistics 15 (2), 2003, pp. 6-10. (in Chinese)

4. Lu, Y.-G., Jiang, D.-W., Jiang, D.-J., "Study and 3D simulation of KE-rod directional dispense", Journal of Ballistics 14 (1), 2002, pp. 27-31. (in Chinese)

5. Jiang, J.-W., Men, J.-B., Lu, Y.-G., Jiang, D.-J., "Numerical simulation of KE-rod directional disperse", Explosion and Shock Waves 24 (1), 2004, pp. 85-89. (in Chinese)

6. Octavio, Melchor-Lucero, P. E., M. ASCE, Cesar J. Carrasco, A. M ASCE, et al., "Computer modeling for a generalized approach to measure impact damage", Journal of Engineering Mechanics 133 (3), 2007, pp. 299-307.

7. Chocron, S., Anderson, C., E., Walker, J. D., "A Unified Model for Long-rod Penetration in Multiple Metallic Plate", International Journal of Impact Engineering 28 (4), 2003, pp. 391-411.

8. Anderson, C., E, Walker, J, D, Bless, S, J, "On the L/D Effect for Long-rod Penetration", International Journal of Impact Engineering 18 (3), 1996, pp. 247-264.

9. Johnson, G., R., Cook, W., H, "Lagrangian EPIC Code Computations for Oblique, Yawed-rod Impacts onto Thin-plates and Spaced-plate Targets at Various Velocities", International Journal of Impact Engineering 14 (1-4), 1993, pp. 373-383.

10. Gee, D., J., Littlefield, D., L, "Yaw Impact of Rod Projectiles", International Journal of Impact Engineering 26 (1-10), 2001, pp. 211-220.

11. Chen, X.-W., Li, Q.-M, "Transition from Non-deformable Projectile Penetration to Semi-hydrodynamic Penetration", Journal of Engineering Mechanics 130 (1), 2004, pp. 123-127.
12. LS-DYNA, "Keyword User's Manual", Version 970, Livermore Software Technology Corporation: Livermore, California, 2003.

13. Lu, Y.-G., "Study of terminal ballistics of KE-rod", China Academy of Engineering Physics, Chengdu, 2002, pp. 2-3. (in Chinese)

14. Qian, L.-X., "Study on damage effect of dense fragment cluster impact on thin target", China Academy of Engineering Physics, Chengdu, 2002, pp. 4-19. (in Chinese)

15. Wang, S.-S., Li, C.-J., Ma, X.-F., "An experimental study on the initiation of covered charge impacted by tungsten alloy fragments", Acta Armamentarii 22 (2), 2001, pp. 189-191. (in Chinese)

16. S., J., Brown, "Energy release protection for pressurized system. Part II: Review of studies into impact/terminal ballistics", Applied Mechanics Reviews 39 (2), 1986, pp. 177-201.

17. Lu, Y.-G., Yang, S.-Q., "The Analytical Model of KE-rod Piercing Armor Based on THOR Formula", Journal of Projectiles, Rockets, Missiles and Guidance 25 (1), 2005, pp. 27-30. (in Chinese)

18. Liu, T., "The Lethality Assessment Method of Air-Defense Missile Warhead", Nanjing University of Science and Technology, Nanjing, 2004, pp. 53-55. (in Chinese)

19. Lu, Y.-G., "Lethality Assessment Method of KE-rod Anti-TBM Warhead Based on Virtual Model", China Academy of Engineering Physics, Chengdu, 2004, pp. 18-24. (in Chinese)

20. Lu, Y.-G., "The Numerical Investigation on the Destructive Effect of KE-rods Impacting Metallic Targets", China Academy of Engineering Physics, Chengdu, 2004, pp. 6-9. (in Chinese)

21. John, G, R, Cook, W, H, "Fracture Characteristics of Three Metals Subjected to Various Strains, Strain Rates, Temperatures and Pressures", Engineering Fracture Mechanics 21 (1), 1985, pp. 3148.

22. Walsh, J., M., Rice, M., H., McQueen, R, G., Yarger, F., L., "Shock-wave compressions of twenty-seven metals: equation of state of metals", Physics Review 108 (2), 1957, pp. 196-216. 